\title{
Theoretical Study on Proton Transfer and Energetics in Ammonium Nitrate $\left(\mathrm{NH}_{4} \mathrm{NO}_{3}\right)_{\mathrm{n}}$ Cluster System
}

\author{
Jo Takeuchi and Kyozaburo Takeda
}

\begin{abstract}
We study the mechanism and energetics of proton transfer (PT) in ammonia-nitric acid systems by employing $a b$ initio calculations. PT does not occur in the single ammonium nitrate unit $\left(\mathrm{NH}_{3}-\mathrm{HNO}_{3}\right.$; monomer) without the application of an external electric field. In contrast, PT is energetically favorable in oligomers $\left(\mathrm{NH}_{4} \mathrm{NO}_{3}\right)_{\mathrm{n}=2-8}$ due to the internal polarization - resulting in the ammonium and nitrate ions, $\mathrm{NH}_{4}{ }^{+} \cdots \mathrm{NO}_{3}{ }^{-}$. Additionally, we study the energetics of ammonium nitrate oligomerization using simple energy decomposition. The classical electrostatic interaction plays a principal role in the studied oligomerization, whereas the charge transfer interaction is not significant. Consequently, PT in the ammonium nitrate oligomer system enhances the internal electrostatic interaction, resulting in an ionic form with remarkable stability even in the gas phase.
\end{abstract}

Index Terms-Ab initio calculation, ammonium nitrate oligomers, energetic stabilization, proton transfer.

\section{INTRODUCTION}

Ammonium nitrate $\left(\mathrm{NH}_{4} \mathrm{NO}_{3}\right)$ crystallizes in several polymorphic forms, which consists of $\mathrm{NH}_{4}{ }^{+}$and $\mathrm{NO}_{3}{ }^{-}$ions [1]-[6]. The room-temperature phase of ammonium nitrate is designated as phase IV, which is stable between 255 and 305 $\mathrm{K}$ [1]. On the other hand, ammonium nitrate has been identified in atmospheric aerosol as one of the typical air pollutants. In gas phase, nitric acid and ammonia react to form ammonium nitrate aerosol particles; this gas-particle conversion has been frequently examined in studies on atmospheric observation [7]. Thermodynamic models have revealed that solid ammonium nitrate forms in the atmosphere, even at low (ppb level) concentrations of ammonia and nitric acid [8]. The ammonium nitrate crystal in atmospheric aerosol particles is also identified as phase IV [9]. However, little is known about the mechanisms of the cohesion of ammonium nitrate. It has been theoretically determined that proton transfer occurs and hydrogen bonds are formed between a pair of ammonium nitrate units (dimer), but not in a single unit (monomer) [10]-[13]. The monomer of ammonia and nitric acid only exists as a hydrogen-bonded $\mathrm{NH}_{3}-\mathrm{HNO}_{3}$ complex, instead of an ionic $\mathrm{NH}_{4} \mathrm{NO}_{3}$ molecule. Since the solid ammonium nitrate crystal consists of only $\mathrm{NH}_{4}{ }^{+}$and $\mathrm{NO}_{3}{ }^{-}$ions, proton transfer from nitric acid to ammonia is expected to play an important role in the cohesion of ammonium nitrate.

Manuscript received October 30, 2013; revised December 30, 2013.

Jo Takeuchi and Kyozaburo Takeda are with the Department of Electrical Engineering and Bioscience, School of Advanced Science and Engineering, Waseda University, Shinjuku, Tokyo 169-8555, Japan, and the first author is also with the Department of Human Science, Takachiho University, Suginami, Tokyo 168-8508, Japan (e-mail: takeuchi-j@takachiho.ac.jp).
In this work, we studied how proton transfer occurs in the ammonium nitrate oligomers by ab initio calculation as the first step forwards the future systematic understanding of the gas-particle conversion in an ammonia-nitrate acid system, that is, the formation of air pollutants. In particular, we focused on the occurrence of proton transfer and the contribution of the intermolecular interaction to energetic stabilization of the system.

\section{Calculation Methodology}

All the $a b$ initio calculations presented herein were performed on the basis of the density functional theory (DFT) at the B3LYP/6-311++G(d,p) level, using the GAUSSIAN 09 program package [14]-[16]. Geometric optimization was performed to obtain global minimum energy structures using different initial geometries and vibrational frequency analysis. The DFT method gives good agreement with the MP2 calculations for structural parameters in ammonium nitrate monomer and dimer [12]. The basis set superposition error (BSSE) was previously estimated to be small (less than $0.4 \mathrm{kcal} / \mathrm{mol}$ ) in monomer and dimer systems when using the 6-311++G( $(d, p)$ basis [11], [12]. Contracted atomic charges from natural bond orbital (NBO) analysis were used to calculate classical Coulomb force for electric field intensities and electrostatic interaction [17]. We attempted a simple energy decomposition analysis (EDA) for interaction energy between molecules. In this work, the intermolecular interaction energy $E_{\text {inter }}$ is defined as

$$
E_{\text {inter }}=E_{\text {total }}^{\text {oligo }}-E_{\text {total }}^{\text {def }},
$$

where $E_{\text {total }}^{\text {def }}$ and $E_{\text {total }}^{\text {oligo }}$ correspond to the sum of separately calculated total energy on deformed molecules and the total energy on an oligomer, respectively. Also, the intermolecular interaction energy $E_{\text {inter }}$ is defined as

$$
E_{\text {inter }}=E_{E S}+E_{C T}+E_{\text {other }},
$$

where $E_{E S}, E_{C T}$, and $E_{\text {other }}$ correspond to electrostatic (ES) energy, charge transfer (CT) energy, and those remainder ( $E_{\text {inter }}$ in (1) $\left.-E_{\mathrm{ES}}-E_{\mathrm{CT}}\right)$, respectively. The $E_{\mathrm{ES}}$ in (2) is calculated as

$$
E_{E S}=E_{E S}^{\text {oligo }}-E_{E S}^{\text {iso }},
$$

where $E_{E S}{ }^{i s o}$ and $E_{E S}{ }^{\text {oligo }}$ correspond to the sum of electrostatic energy on isolated molecules and the electrostatic energy on an oligomer, respectively. Considering no charge transfer between molecules, the $E_{E S}{ }^{\text {oligo }}$ is calculated using the NBO charges of isolated molecules. The $E_{\mathrm{CT}}$ in (2) represents 
resonance-type delocalization interactions between occupied orbitals of one molecule and unoccupied orbitals of the other; the energetic stabilization due to donor-acceptor interactions is estimated by second-order perturbation theory [18]. Considering the precise EDA methods [19], [20], the $E_{\text {other }}$ is supposed to include polarization energy and electronic exchange repulsion energy although they are undivided in this simple method. In ammonium nitrate oligomers, the formation of hydrogen bonds is an important factor to stabilize molecular structures, and the main effects of hydrogen bonds are CT and ES. Thus, this simple method is useful enough to understand the magnitude of quantum-mechanical CT effect vs classical ES effect on the energetic stabilization of intermolecular interaction. The cohesive energy $E_{\text {cohe }}$ is calculated as

$$
E_{\text {cohe }}=E_{\text {total }}^{\text {oligo }}-n E_{\text {total }}^{\text {iso }}
$$

where $E_{\text {total }}^{\text {iso }}, E_{\text {total }}$ oligo , and $n$ correspond to the sum of total energy on isolated $\mathrm{NH}_{3}$ and $\mathrm{HNO}_{3}$ molecules, the total energy on an oligomer, and the number of monomers in an oligomer, respectively.

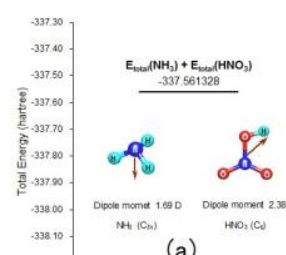

(a)

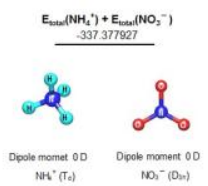

(b)

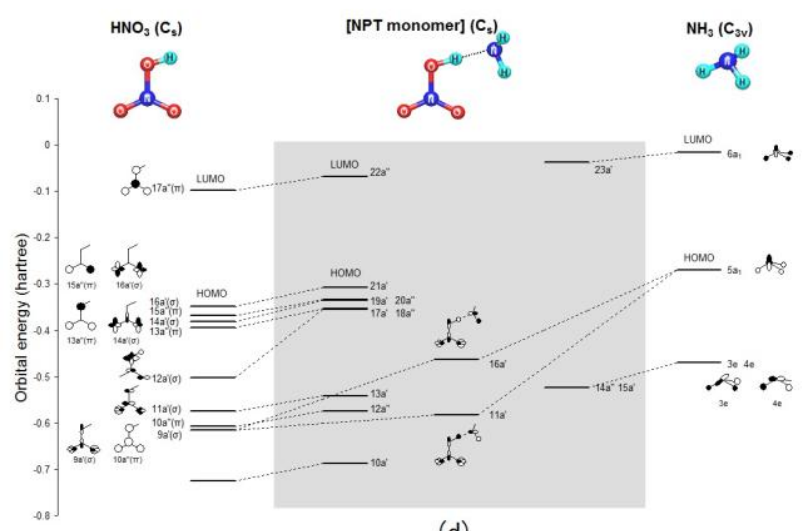

(d)

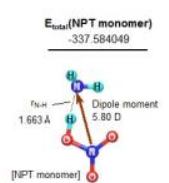

(c) ammonium nitrate monomer. Here, we carried out $a b$ initio calculations on the monomer. The global-minimum structure of the monomer, non-proton-transferred monomer (NPT monomer), is shown in Fig. 1(c). In Fig. 1(c), the nitrogen atom in ammonia and hydrogen atom in nitric acid are most stable at the interatomic distance $\mathrm{r}_{\mathrm{N}-\mathrm{H}}$ of $1.663 \AA$, and the hydrogen bond between ammonia and nitric acid is formed. The total energy of the NPT monomer is lower than the sum of total energies of ammonia and nitric acid, although proton transfer does not occur in the NPT monomer. Here, considering the electronic structure of NPT monomer in Fig. $1(\mathrm{~d})$, the highest occupied molecular orbital (HOMO) $5 \mathrm{a}_{1}$ in ammonia mixes with the energy level $9 \mathrm{a}^{\prime}$ in nitric acid. This orbital mixing causes the formation of a hydrogen bond between ammonia and nitric acid, which contributes to the overall energetic stabilization of the NPT monomer electronic system.

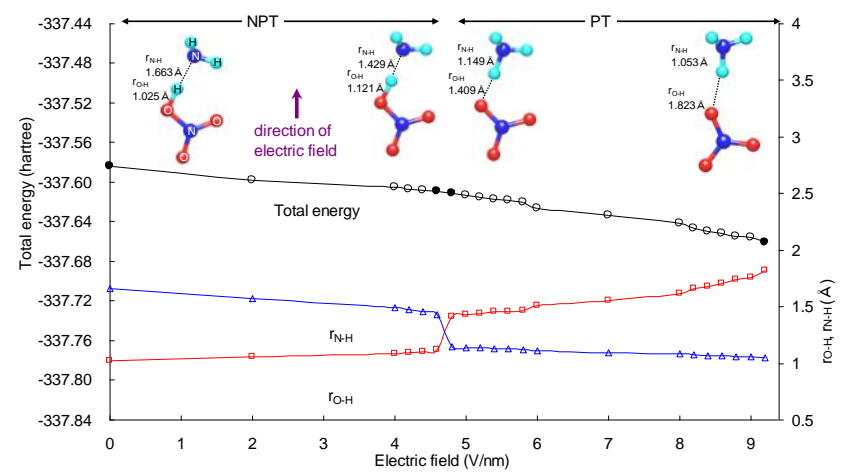

Fig. 2. Changes in the total energies, the interatomic distances $\mathrm{r}_{\mathrm{O}-\mathrm{H}}$ and $\mathrm{r}_{\mathrm{N}-\mathrm{H}}$ and the molecular structures of the monomer as a function of external electric field intensity.

\section{B. Proton Transfer in Ammonium Nitrate Monomer under an External Electric Field}

In the next step, we carried out ab initio calculations of the monomer under an external electric field, as shown in Fig. 2. The direction of an electric field was applied parallel to the dipole moment of the monomer. With an electric field over $4.8 \mathrm{~V} / \mathrm{nm}$, proton transfer spontaneously occurs in the monomer as the total energy becomes lower than that of the NPT monomer system. When the electric field intensity exceeds $9.2 \mathrm{~V} / \mathrm{nm}$, the monomer separates into $\mathrm{NH}_{4}^{+}$and $\mathrm{NO}_{3}{ }^{-}$ions, and the overall system becomes increasingly stable with greater distance between the $\mathrm{NH}_{4}{ }^{+}$and $\mathrm{NO}_{3}{ }^{-}$ions.

Thus, these results show that the existence of an electric field acting on the NPT monomer promotes the occurrence of proton transfer and stabilizes the total energy of the system. The external electric field should fall in the range between 4.8 and $9.2 \mathrm{~V} / \mathrm{nm}$.

\section{Molecular Structures of Ammonium Nitrate Oligomers}

We carried out $a b$ initio calculations on the ammonium nitrate oligomers, $\left(\mathrm{NH}_{4} \mathrm{NO}_{3}\right)_{\mathrm{n}=2 \sim 8}$. The global-minimum structures of the oligomers are shown in Fig. 3. In the optimizing calculations for oligomers, we added a "NPT" monomer to pre-calculated oligomer structure as an initial structure. However, all the oligomers prefer proton-transferred (PT) structures. The electric field intensities acted on the added monomers from the remaining oligomers are also shown in Fig. 3. As we expected, the 
intensities are between 8.16 and $8.77 \mathrm{~V} / \mathrm{nm}$. These results are consistent with the finding that proton transfer occurs in the monomer under an external electric field between 4.8 and 9.2 $\mathrm{V} / \mathrm{nm}$. Therefore, ammonium nitrate oligomers form PT structure due to the effect of their self-induced inner electric field, whereas each monomer should prefer NPT structure if they are separated. It is very interesting that ammonium nitrate oligomers have ionic structures in spite of being in gas phase.

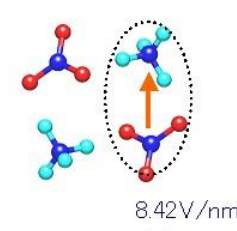

Dimer (2)

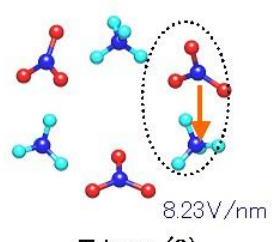

Trimer (3)

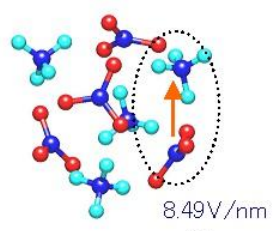

Tetramer (4)

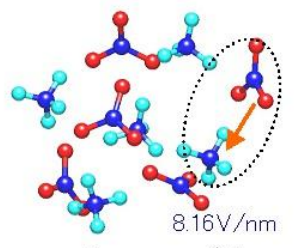

Pentamer (5)

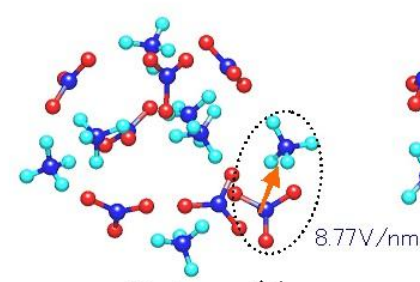

Heptamer (7)

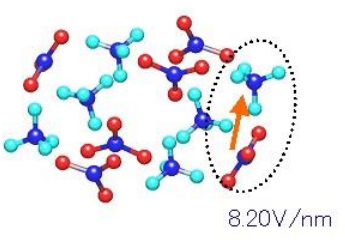

Hexamer (6)

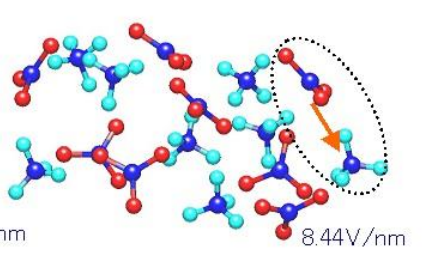

Octamer (8)
Fig. 3. The molecular structures of ammonium nitrate oligomers. The electric field intensities acted on the added monomers from the remaining oligomers.

\section{Energetic Stabilization}

We calculate the cohesive energy, and interaction energies between molecules or ions on the ammonium nitrate monomer and oligomers, as shown in Fig. 4. The energies are standardized relative to the formula unit, $\mathrm{NH}_{4} \mathrm{NO}_{3}$.

The cohesive energy $E_{\text {cohe }}$ is gradually increasing with the increased size of oligomers. This result infers that ammonium nitrate oligomer could easily grow in size. As mentioned in the previous discussion, the oligomers, $\left(\mathrm{NH}_{4} \mathrm{NO}_{3}\right)_{\mathrm{n}=2 \sim 8}$, prefer the PT structures (ionic structures). As a result, the electrostatic energy $E_{\mathrm{ES}}$ of the oligomers largely contributes to the energetic stabilization. Since the even-numbered oligomers easily form alternant arrangements of positive and negative ions, the $E_{\mathrm{ES}}$ of the even-numbered oligomer is larger than that of the adjacent odd-numbered. The $E_{\mathrm{ES}}$ increases with the increased size of the oligomers because the electrostatic interactions stabilize the system even at a large distance. Although the charge transfer energy $E_{\mathrm{CT}}$ also contributes to the energetic stabilization, the contribution is not as large as that of the $E_{\mathrm{ES}}$. In contrast to the $E_{\mathrm{ES}}$, the $E_{\mathrm{CT}}$ of the odd-numbered oligomer is larger than that of the adjacent even-numbered. The hydrogen bonds in the oligomers are formed due to the charge transfer from the oxygen atoms in the nitrate group to hydrogen atoms in the ammonium group. Here, the interatomic distance $\mathrm{r}_{\mathrm{O}-\mathrm{H}}$ in hydrogen bonds of each oligomers is shown in Fig. 5. The changes of the average distances of $\mathrm{r}_{\mathrm{O}-\mathrm{H}}$ in Fig. 5 are rather similar to those in the $E_{\mathrm{CT}}$ in Fig. 4. Thus, the changes in the $E_{C T}$ show the dependency on the interatomic distance $\mathrm{r}_{\mathrm{O}-\mathrm{H}}$. We can not provide the physical interpretation for the $E_{\text {other }}$ because that is defined as a reminder, as mentioned in Section II.

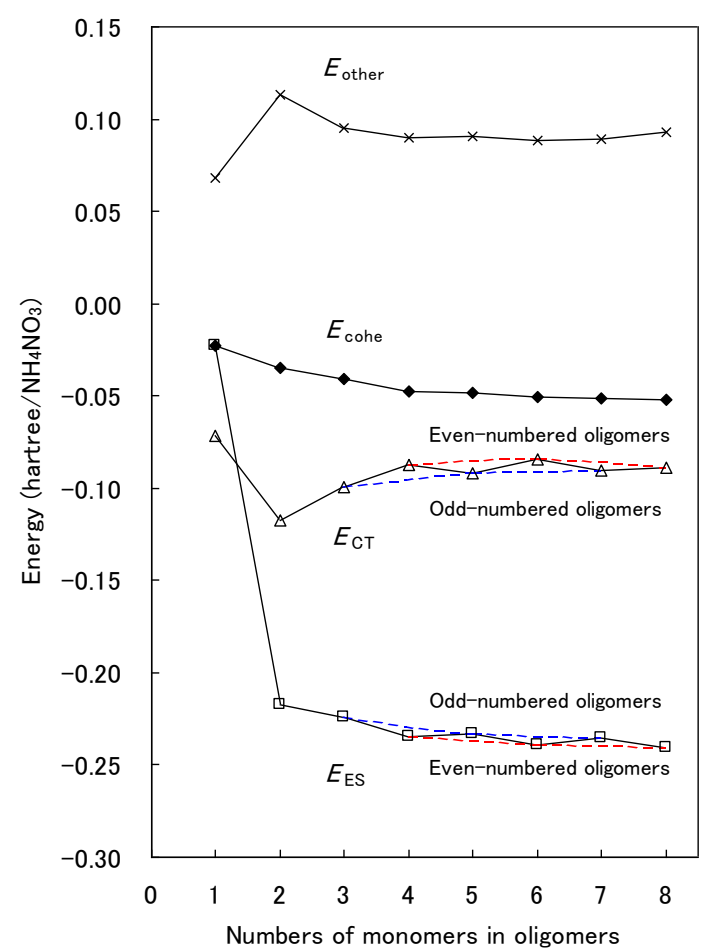

Fig. 4. Changes in the cohesive energy $E_{\text {cohe }}$, electrostatic energy $E_{\mathrm{ES}}$, charge transfer energy $E_{\mathrm{CT}}$, and those remainder in interaction energy $E_{\text {other }}$ of each oligomer as a function of the number of monomers in oligomers. The energies are standardized relative to the formula unit, $\mathrm{NH}_{4} \mathrm{NO}_{3}$.

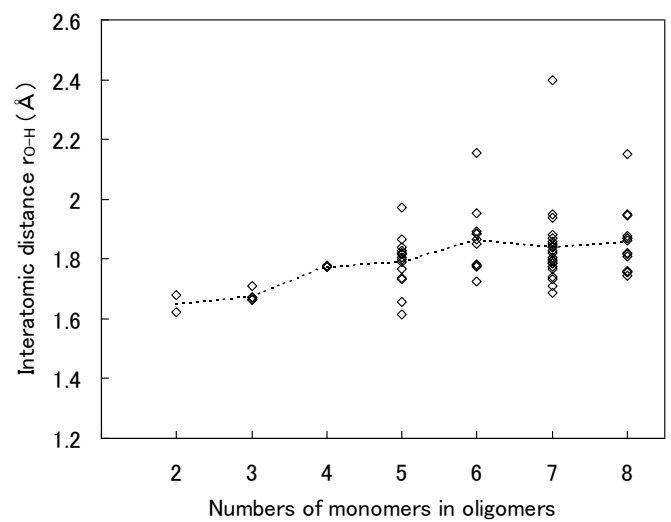

Fig. 5. Changes in the interatomic distance $\mathrm{r}_{\mathrm{O}-\mathrm{H}}$ in hydrogen bonds of the oligomers as a function of the number of monomers in oligomers. Dotted line refers the average distances of the $\mathrm{r}_{\mathrm{O}-\mathrm{H}}$.

\section{CONCLUSION}

We have studied the mechanism and energetics of proton transfer in ammonia-nitric acid systems by $a b$ initio calculation at the B3LYP/6-311++G(d,p) level. We demonstrate that PT does not occur in the single ammonium nitrate unit $\left(\mathrm{NH}_{3}-\mathrm{HNO}_{3}\right.$; monomer) without the application of an external electric field whereas PT is energetically favorable in oligomers $\left(\mathrm{NH}_{4} \mathrm{NO}_{3}\right)_{\mathrm{n}=2 \sim 8}$ due to the internal polarization - resulting in the ammonium and nitrate ions, $\mathrm{NH}_{4}{ }^{+} \cdots \mathrm{NO}_{3}{ }^{-}$. Accordingly, the classical electrostatic interaction is enhanced and plays a principal role in the oligomerization, whereas the charge transfer interaction is 
not significant. Consequently, this internal electrostatic interaction caused by PT in the ammonium nitrate oligomer system results in an ionic form with remarkable stability even in the gas phase.

\section{ACKNOWLEDGMENT}

K.Takeda thanks Dr. Gareth Griffiths of Cavendish Laboratory, University of Cambridge for fruitful discussion on ammonia compounds. The computations in this work were partly performed using Research Center for Computational Science, Okazaki, Japan.

\section{REFERENCES}

[1] C. S. Choi, J. E. Mapes, and E. Prince, "The structure of ammonium nitrate (IV)," Acta Crystallogr. Sect. B, vol. 28, pp. 1357-1361, May 1972.

[2] S. Yamamoto and Y. Shinnaka, "X-ray study of polyorientational disorder in cubic $\mathrm{NH}_{4} \mathrm{NO}_{3}$," J. Phys. Soc. Jpn., vol. 37, pp. 724-732, Sep. 1974.

[3] S. Swaminathan, "A probable crystallographic path for phase transformations in single crystals of ammonium nitrate," Acta Crystallogr. Sect. A, vol. 32, pp. 499-504, May 1976.

[4] B. W. Lucas, M. Ahtee, and A. Hewat, "The crystal structure of phase II ammonium nitrate," Acta Crystallogr. Sect. B, vol. 35, pp. 1038-1041, May 1979.

[5] B. W. Lucas, M. Ahtee, and A. Hewat, "The structure of phase III ammonium nitrate," Acta Crystallogr. Sect. B, vol. 36, pp. 2005-2008, Sep. 1980.

[6] M. Ahtee, K. L. Smolander, B. W. Lucas, and A. Hewat, "The structure of the low-temperature phase $\mathrm{V}$ of ammonium nitrate, $\mathrm{ND}_{4} \mathrm{NO}_{3}$, , Actc Crystallogr. Sect. C, vol. 39, pp. 651-655, June 1983.

[7] J. H. Seinfeld and S. N. Pandis, Atmospheric Chemistry and Physics, 2nd ed., New York: Wiley, 2006, pp. 472-478.

[8] A. W. Stelson and J. H. Seinfeld, "Relative humidity and temperature dependence of ammonium nitrate dissociation constant," Atmos. Environ., vol. 16, pp. 983-992, 1982.

[9] R. M. Harrison and W. T. Sturges, "Physico-chemical speciation and transformation reactions of particulate atmospheric nitrogen and sulphur compounds," Atmos. Environ., vol. 18, pp.1829-1833, 1984.
[10] Z. Latajka, M. M. Szczçsniak et al., "Properties of strong hydrogen-bonded systems. II. Ab intio SCF-MO of the hydrogen bond between nitric acid and ammonia," J. Comput. Chem., vol. 1, pp. 417-419, 1980.

[11] M.-T. Nguyen, A. J. Jamka, R. A. Cazar, and F.-M. Tao, "Structure and stability of the nitric acid-ammonia complex in the gas phase and in water," J. Chem. Phys., vol. 106, pp. 8710-8717, June 1997.

[12] S. Alavi and D. L. Thompson, "Theoretical study of proton transfer in ammonium nitrate clusters," J. Chem. Phys., vol. 117, pp. 2599-2608, Aug. 2002.

[13] J. Takeuchi, Y. Masuda, R. Clark, and K. Takeda, "Theoretical studies on proton transfer in ammonium nitrate monomer and dimer," Jpn $J$. Appl. Phys., vol. 52, 076302, June 2013.

[14] C. Lee, W. Yang, and R. G. Parr, "Development of the Colle-Salvetti correlation-energy formula into a functional of the electron density," Phys. Rev. B, vol. 37, pp. 785-789, Jan. 1988.

[15] A. D. Becke, "Density-functional thermochemistry. III. The role of exact exchange," J. Chem. Phys., vol. 98, pp. 5648-5652, April 1993.

[16] Gaussian 09, revision A.1, Gaussian, Inc., Wallingford, 2009.

[17] A. E. Reed, R. B. Weinstock, and F. Weinhold, "Natural population analysis," J. Chem. Phys., vol. 83, pp. 735-746, July 1985.

[18] A. E. Reed, L. A. Curtiss, and F. Weinhold, "Intermolecular interactions from a natural bond orbital, donor-acceptor viewpoint," Chem. Rev., vol. 88, pp. 899-926, Sep. 1988.

[19] K. Kitaura, and K. Morokuma, "A new energy decomposition scheme for molecular interactions within the Hartree-Fock approximation," Int J. Quantum Chem., vol. 10, pp. 325-340, 1976.

[20] P. Su and H. Li, "Energy decomposition analysis of covalent bonds and intermolecular interactions," J. Chem. Phys., vol. 131, July 2009.

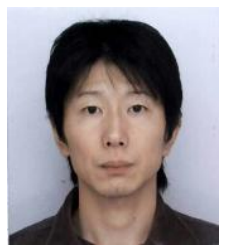

J. Takeuchi took the degree of M.A. in engineering at Waseda University, Japan. He is in the doctoral course, School of Advanced Science and Engineering, Waseda University. Also, he is the assistant professor of the Department of Human Science, Takachiho University, Japan.

K. Takeda is the professor of the Department of Electrical Engineering and Bioscience, School of Advanced Science and Engineering, Waseda University, Japan. 